\title{
VARIACION DE LA DENSIDAD POBLACIONAL DE SMINTHURUS VIRIDIS (L.) (COLLEMBOLA: SMINTHURIDAE) EN LA IX REGION DE LA ARAUCANIA, CHILE
}

\author{
POPULATION DENSITY VARIATION OF SMINTHURUS VIRIDIS (L.) \\ (COLLEMBOLA: SMINTHURIDAE) IN THE IX REGION OF \\ LA ARAUCANIA, CHILE
}

\author{
Carlos Klein ${ }^{1}$, Ramón Rebolledo ${ }^{1}$, Claudia Cerda ${ }^{1}$ \& Alfonso Aguilera ${ }^{1,2}$ \\ ${ }^{1}$ Facultad de Ciencias Agropecuarias y Forestales. Universidad de La Frontera. \\ Casilla 54-D, Temuco, Chile.Email: cklein@ufro.cl \\ ${ }^{2}$ Centro Regional de Investigación Carillanca del Instituto de Investigaciones Agropecuarias. Temuco, Chile.
}

\begin{abstract}
RESUMEN
La "pulga saltona" o "colémbolo de la alfalfa" S. viridis (L.) es una plaga secundaria de reciente introducción a Chile que alcanzado cierta importancia en praderas de alfalfa en la zona centro-sur del país. El estudio se llevó a cabo en alfalfares de la ex estación experimental Maipo de la Universidad de La Frontera en la zona agroecológica del llano central y en el INIA Carillanca correspondiente a la precordillera. En ambas zonas se realizó un muestreo cada 15 días con red entomológica durante el período de junio de 1996 a marzo de 1997 con el objetivo de determinar la fluctuación poblacional, además de sus eventuales enemigos naturales. Los resultados demuestran que la población fue muy baja en el llano central de octubre a marzo y se mantuvo baja en los meses de junio a agosto para llegar a un máximo de 14,3 individuos por $\mathrm{m}^{2}$ en septiembre. En la precordillera ocurrieron dos aumentos poblacionales, ambos en la segunda quincenas de junio y julio, bajando en septiembre. Además, los máximos se obtuvieron en octubre y noviembre llegando a 15,9 individuos por $\mathrm{m}^{2}$. Durante los meses de enero y febrero no se logró colectar ejemplares. Se encontró una significativa relación entre la temperatura y altura de la planta con la fluctuación poblacional en el llano central, no así en la precordillera. El único enemigo natural encontrado en ambas zonas agroecológicas durante el mes de enero, alimentándose cuando el colémbolo se encuentra en diapausa estival, fue el chinche Nabis punctipennis (Blanchard).
\end{abstract}

Palabras Claves: Sminthurus viridis, fluctuación poblacional, Nabis punctipennis, La Araucanía, Chile.

\section{ABSTRACT}

The lucerne flea S. viridis (L.) is a secondary pest of recent introduction to Chile that has archevied more importance in lucerne in the central-south part of the country. The study was conducted in the former experimental station Maipo of the University of La Frontera in Temuco, in the agroecological area of the central plain, and in the Regional Research Center INIA Carillanca in the preandean area. The population variation was studied in both areas using a standard entomological net every 15 days in the period from june 1996 to march 1997, as well as the eventual natural enemies. The results shows in the central plain a low population from june to august, but an increase up to 14,3 individual per $\mathrm{m}^{2}$ in september, declining strongly from october to march. In the preandean area two peaks occurred, both during the second halfs of october to november with a peak of 15,8 individual per $\mathrm{m}^{2}$. During the summer months january to february no specimens were collected. The relation between the population variation and the size of lucerne was statistically significance in the central plain, but not in the preandean area. The only natural enemie found was the bug Nabis punctipennis (Blanchard) feeding on $S$. viridis, when this species was in summer diapause.

Keywords: Sminthurus viridis, population variation, Nabis punctipennis, La Araucanía Region, Chile. 


\section{INTRODUCCION}

La "pulga saltona" o "colémbolo de la alfalfa", Sminthurus viridis (Linnaeus), está ampliamente distribuida en el mundo asociada a una diversidad de plantas, especialmente alfalfa y praderas artificiales de trébol, donde constituye una plaga de importancia económica en Australia y algunos países de Europa (Gillot 1980, Pedigo1989, Borror et al. 1989, Greenslade 1991). Su nombre común se debe a que son capaces de saltar verticalmente hasta unos $30 \mathrm{~cm}$ y pueden alcanzar velocidades de $1,4 \mathrm{~m} / \mathrm{seg}$.

En Chile este colémbolo se manifiesta con mayor frecuencia en alfalfa sin que sus poblaciones hayan llegado, hasta la fecha, a constituir un nivel de plaga que signifique su control (González 1989, Aguilera \& Gerding 1990, Aguilera 1992, Klein \& Waterhouse 2000). Fue detectada entre las regiones VII y X (Cisternas et al 1990, Cisternas 1994) y ha sido observada causando daños en lupino (Aguilera et al. 1996). Se han encontrado en Chile diversos enemigos naturales, principalmente depredadores, que contribuyen al control natural de sus poblaciones (Prado 1991, Artigas 1994). En otros países se citan a diversos artrópodos depredadores de la pulga saltona de la alfalfa (Greenslade 1991) tales como coleópteros carábidos y estafilínidos, hormigas, arañas, pseudoescorpiones, ciempiés y ácaros en el control natural de Sminthurus viridis. La duración del ciclo de vida varía de 51 a 74 días a temperaturas de 13 y $17^{\circ} \mathrm{C}$, respectivamente (Cisternas et al. 1990, citando a Maglagan 1932). Richards y Davies (1984) consideran que en Inglaterra las condiciones óptimas para su desarrollo ocurren con una humedad cercana a la saturación y una temperatura aproximada a los $13^{\circ} \mathrm{C}$, pudiendo llegar a vivir 75 días y tener cinco generaciones anuales.

La dinámica poblacional de $S$. viridis está influenciada no solamente por sus enemigos naturales, sino también por el comportamiento alimenticio, tipo de suelo y composición botánica (Cisternas et al. 1990). Cuando el verano se presenta cálido y seco, los ataques se concentran en otoño y primavera siguientes (Artigas 1994)

El presente trabajo tuvo como objetivo estudiar algunos puntos de la dinámica poblacional de Sminthurus viridis sobre alfalfa en el llano central y precordillera de La Araucanía, considerando las variables de temperatura, humedad relativa y altura de la planta, además de la determinación de sus enemigos naturales (depredadores o parasitoides)

\section{MATERIALES Y METODOS}

Los estudios se llevaron a cabo en la ex estación experimental Maipo perteneciente a la Universidad de La Frontera, ubicada en el sector urbano de la ciudad de Temuco a 100 m.s.n.m., (HUSO 18H0704562; UTM 5708582 medidas con GPS marca Garlin modelo III Plus) correspondiente a la zona agroecológica del llano central, y en el centro de Investigación INIA Carillanca en la comuna de Vilcún (HUSO 18H07246698; UTM5713954 a 200 m.s.n.m.) en la precordillera. Ambas zonas agroecológicas se encuentran en la IX Región de La Araucanía y fueron descritas por Rouanet (1983 a, b).

Los ensayos en el llano central se realizaron en cuatro hectáreas de alfalfa (cultivar WL-320), establecido en octubre 1993 y que sufre latencia invernal. La pradera se manejó sin insecticidas ni riego, pero se realizó control de malezas regularmente con herbicida (Paraquat) en invierno. Los cortes para heno se efectuaron entre octubre y febrero, con pastoreo directo de vacunos en abril.

Los estudios en la precordillera se realizaron en cuatro hectáreas de alfalfa con un cultivar similar al anterior, establecida en octubre 1994. Se realizaron fertilizaciones sucesivas en los años siguientes, agregando estiércol. Desde su establecimiento y hasta la ejecución del presente trabajo no se aplicaron insecticidas, pero sí un herbicida (Glifosato) todos los inviernos. Se practicó riego por tendido desde octubre hasta el término del verano. La pradera se manejó de distintas formas, incluyendo pastoreo, en años anteriores al ensayo, henificación, silo y soiling. En ambas zonas agroecológicas los muestreos se realizaron cada 15 días, desde junio 1996 hasta marzo 1997, mediante una red entomológica de $30 \mathrm{~cm}$ de diámetro con la cual se efectuaron 10 golpes de $180^{\circ}$ a paso regular sin cambiar al operario. Considerando la dimensión de las parcelas, éstas se dividieron en cuatro cuadrantes de una hectárea cada uno para hacer más representativo el muestreo, lo que permitió, además, calcular la abundancia relativa por $\mathrm{m}^{2}$. En cada muestreo se ingresaba a un cuadrante distinto (1 hectárea), en el cual se trazaba una diagonal imaginaria y se pasaban los golpes de red a través de la línea con tres repeticiones por oportunidad. El material colectado se dispuso en frascos con alcohol $75 \%$ debidamente rotulados.

En ambos sectores se midió periódicamente, cada 
15 días, el estado de crecimiento de la alfalfa en 10 muestras tomadas al azar.

El conteo de $S$. viridis se realizó mediante una lupa e instrumental entomológico apropiado, sin diferenciar entre adultos y ninfas. El diseño experimental correspondió a un muestreo dirigido en dos sectores y para el análisis de datos se empleó la prueba de correlación de rangos de Spearman con una significancia del 5\% (Wayne 1988).

Se realizó una crianza estival en trébol rosado dispuesto en seis jaulas de crianza, en las cuales se colocaron 10 individuos de $S$. viridis en cada una. Las jaulas se mantuvieron en estado de saturación hídrica permanente rociando agua diariamente durante las tres semanas que duró el ensayo para comprobar un eventual estado de diapausa estival y ver, paralelamente, la posible emergencia de algún parasitoide.

\section{RESULTADOS Y DISCUSION}

Abundancia Relativa de SMINTHURUS VIRIDIS

Los resultados obtenidos en el estudio de densidad poblacional en el período de junio 1996 a marzo 1997 significaron una media de 2 individuos $/ \mathrm{m}^{2}$ y 5 indivi- duos $/ \mathrm{m}^{2}$ para las zonas agroecológicas del llano central y precordillera respectivamente. Estas cifras, sin embargo, difieren considerablemente de lo señalado por Greenslade (1991) quien indica que en Australia es posible encontrar 2.000 a 3.000 colémbolos $/ \mathrm{m}^{2}$ en zonas áridas y hasta 100.000 individuos $/ \mathrm{m}^{2}$ en la isla subabtártica de Macquarie, Sin embargo, es necesario hacer hincapié, que esas cifras corresponde a diversas especies de Collembola.

En el llano central el mayor número de individuos se registró el 30 de septiembre con 14,3 individuos $/ \mathrm{m}^{2}$, mientras que en la precordillera alcanzó a 15,9 individuos $/ \mathrm{m}^{2}$ (Figuras 1 y 2 ).

FLUCTUACIÓN POBLACIONAL EN EL LLANO CENTRAL La fluctuación poblacional de $S$. viridis mostró poblaciones notoriamente bajas en los meses de junio a agosto, debido a las condiciones climáticas y a la aplicación de un herbicida que afectó no solamente su base alimenticia sino también su protección. A partir de septiembre ocurrió un notable aumento poblacional con una media de 9,8 individuos por $\mathrm{m}^{2}$ en la primera quincena y 14,3 individuos $/ \mathrm{m}^{2}$ en la segunda quincena del mismo mes, para disminuir a partir de octubre hasta marzo, en que la pulga saltona prácticamente desapareció (Fig. 1).

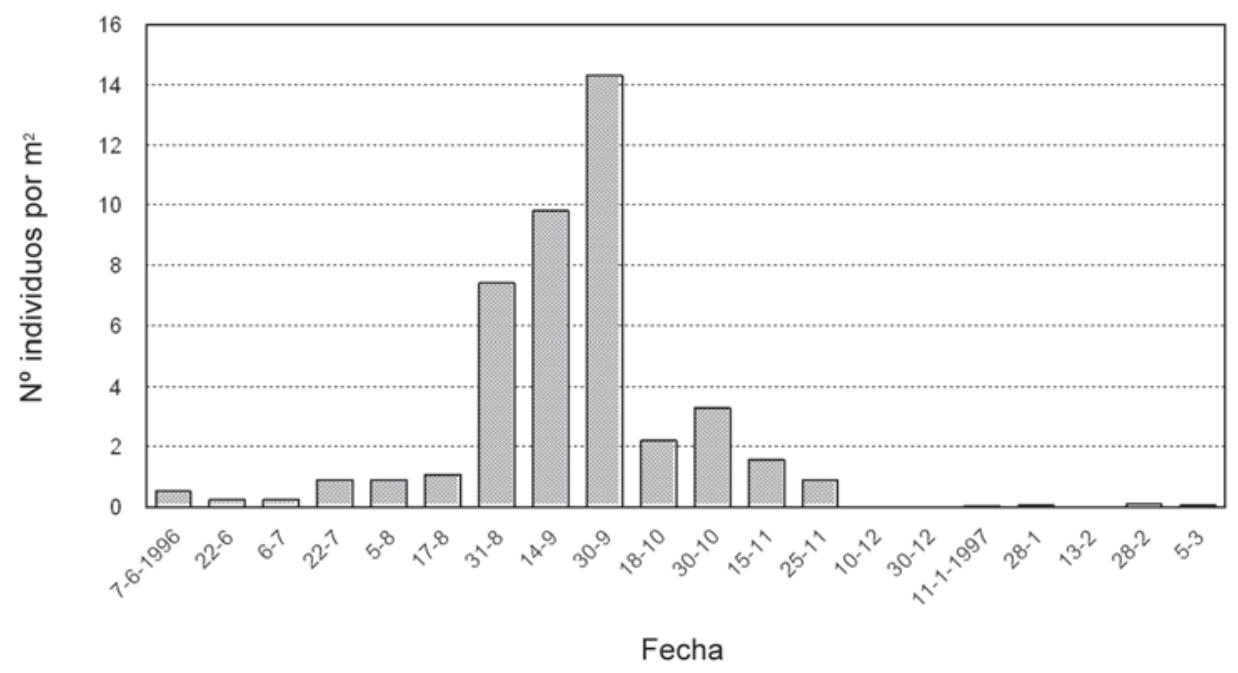

Figura 1. Fluctuación poblacional de Sminthurus viridis en el llano central de La Araucanía (Chile).

Figure 1. Population variation of Sminthurus viridis in the central plain of La Araucanía (Chile). 
Se encontró una relación estadísticamente significativa $(\mathrm{P}<0,05)$ entre la altura de la planta de alfalfa y la fluctuación poblacional. Es decir, a mayor altura mayor número de individuos, siendo estas alturas de 15 hasta $33 \mathrm{~cm}$. El factor altura de la planta constituye un ambiente adecuado para el desarrollo de esta especie y la protege de la desecación con el refugio que le ofrece el follaje. Chinery (1988) y Bonnemaison (1975) señalan la susceptibilidad de la pulga saltona, precisamente, a este factor.

Se encontró una significativa relación negativa entre la temperatura ambiente y la población de $S$. viridis. Sin embargo, la diapausa inducida por el aumento de temperatura, permitió que el chinche depredador sobre las pulgas saltonas inactivas, Nabis punctipennis, enmascarara la influencia de este factor ambiental en la fluctuación poblacional. Maglagan (1932) citado por Cisternas et al. (1990) señala que la temperatura óptima para el desarrollo de S. viridis está en el rango de 13 a $17^{\circ} \mathrm{C}$, mientras Richards y Davies (1984) mencionan como ideal una temperatura $13{ }^{\circ} \mathrm{C}$.
Durante la ejecución del presente trabajo, la temperatura en el llano central osciló entre 4 y $19,2{ }^{\circ} \mathrm{C}$, con un promedio de $13,2^{\circ} \mathrm{C}$ para los diez meses de observaciones. La temperatura media del mes de septiembre se registró en $12,9{ }^{\circ} \mathrm{C}$ y correspondió al mes donde se obtuvo el mayor número de individuos en la colecta. Las temperaturas primaverales y veraniegas más altas coinciden con una fuerte disminución de la población (Fig. 1).

FLUCTUACIÓN POBLACIONAL EN LA PRECORDILLERA En la precordillera la fluctuación poblacional presentó dos aumentos notorios en la segunda quincena de junio y julio, con promedio de 9,3 y 10,3 individuos $/ \mathrm{m}^{2}$ respectivamente, bajando en septiembre debido probablemente a una aplicación de glifosato. Otros dos máximos ocurrieron el 7 de octubre con una media de 14,5 individuos $/ \mathrm{m}^{2}$ y el 4 de noviembre con 15,9 individuos $/ \mathrm{m}^{2}$. En los meses de enero y febrero la población prácticamente desapareció, hasta recuperarse levemente en marzo (Fig. 2).

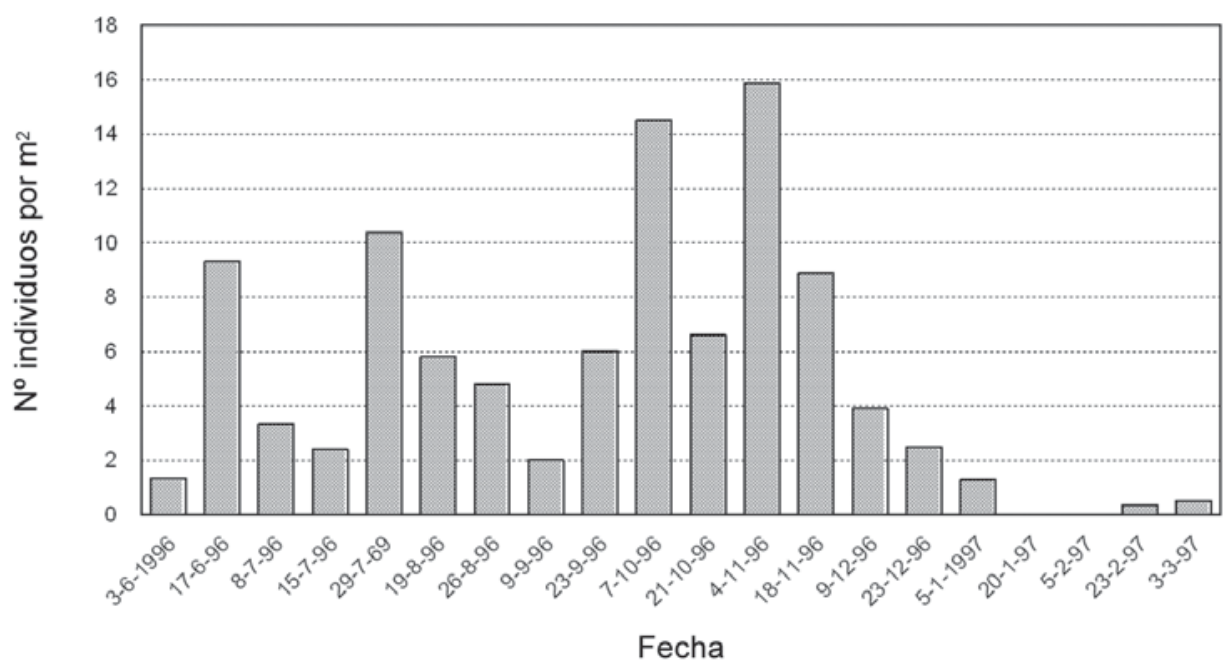

FIgURA 2. Fluctuación poblacional de Sminthurus viridis en la precordillera andina de La Araucanía (Chile).

Figure 2. Population variation of Sminthurus viridis in the preandean zone of La Araucanía (Chile).

A diferencia de lo señalado para el llano central, en esta zona agroecológica no ocurrió una relación significativa entre el factor de altura de planta y fluctuación poblacional, encontrándose el mayor número de individuos a $40 \mathrm{~cm}$ del suelo en noviembre. A su vez, la relación entre la temperatura ambiente y la fluctuación poblacional en esta zona agroecológica no fue un factor estadísticamente significativo $(\mathrm{P}>0,05)$ pese a que la media de temperatura en los diez meses de estudio fue de $11,5^{\circ} \mathrm{C}$. 
La marcada fluctuación poblacional se explica por la dependencia de los colémbolos con la alta humedad. Algunas especies de esta orden tienen asombrosos mecanismos de adaptación para períodos críticos secos que incluyen una aparente antihidrobiosis y una reabsorción de agua de las fecas. Aunque este fenómeno no fue objeto de este estudio, estados juveniles de $S$. viridis pueden alimentarse vorazmente de adultos recién muertos, acumulando de este modo uratos que pueden precipitar su propia desaparición. Este proceso juega un rol importante en la regulación de la densidad poblacional de este insecto (Greenslade 1991).

La baja población en los meses de octubre a marzo se explica en ambas zonas agroecológicas por la diapausa estival, los sucesivos cortes de la alfalfa, lo que favorece una mayor exposición a la desecación, y a los depredadores presentes.

La humedad relativa no fue un factor significativo en la fluctuación poblacional de $S$. viridis en ninguna de las dos zonas agroecológicas estudiadas, pese a que la actividad de los colémbolos y su distribución está fuertemente influenciada por la misma, aunque también pueden ser atraídos por altas concentraciones de dióxido de carbono y otros metabolitos producidos por microorganismos (Greenslade 1991).

\section{Crianza En Verano}

Los resultados de la crianza estival de la pulga saltona indican que en las condiciones del ensayo se produce una diapausa estival, tal como ocurre en otros países (Wallace y Mackerras 1979). Los ejemplares utilizados no mostraron estímulos para saltar, sus movimientos fueron lentos y se mantuvieron en la base de las plantas sin provocar daños, comportamiento también citado por Odum (1972). Se observaron, además, cambios de color desde tonos verdes y verde amarillos a anaranjados y marrón que Phillips (1976) relaciona con los cambios hormonales de una diapausa. Sólo en una de seis jaulas de crianza ocurrió postura de huevos, que no lograron eclosionar adecuadamente.

ENEMIGOS NATURALES

El único depredador de importancia observado consumiendo $S$. viridis desde noviembre hasta febrero fue el nábido Nabis punctipennis (Blanchard) (Hemiptera: Nabidae). Para este depredador la captura de $S$. viridis no ofreció dificultades por la len- titud de movimientos y la disminución de la respuesta a estímulos de la presa en este período. De la crianza estival no se obtuvo ningún parasitoide.

\section{CONCLUSIONES}

En la zona agroecológica del llano central el mayor número de individuos alcanzó a $14,3 / \mathrm{m}^{2}$ en septiembre, mientras que en la precordillera se llegó a un máximo en noviembre con 15,9 individuos $/ \mathrm{m}^{2}$.

En el llano central las fluctuaciones de la abundancia relativa de Sminthurus viridis se afectó claramente por la altura de la planta (cortes realizados) y la temperatura.

En la precordillera, en cambio, no se encontró una relación entre la abundancia relativa de Sminthurus viridis y la altura de planta, temperatura ambiente ni humedad relativa debido posiblemente al diferente manejo realizado a la alfalfal.

El chinche Nabis punctipennis (Blanchard) es el depredador de mayor importancia encontrado en los meses de verano. Finalmente, se comprobó la existencia de diapausa estival de $S$. viridis bajo las condiciones del estudio.

\section{BIBLIOGRAFIA}

Artigas, J. 1994. Entomología Económica. Insectos de interés agrícola, forestal, médico veterinario (nativos, introducidos y susceptibles de ser introducidos). Vol. I. Edic. U. De Concepción, 1.126p.

Aguilera, A. 1992. Insectos y ácaros asociados a la alfalfa en la IX Región de La Araucanía pp. 113-145. En Romero. O. (Ed.). Seminario alfalfa y su utilización en la zona sur. INIA, serie Carillanca $\mathrm{N}^{\mathrm{o}} 31$ (Temuco, Chile), 224p.

Aguilera, A. \& M. Gerding. 1990. Plagas en alfalfa. Seminario producción y utilización de la alfalfa en la zona centro sur. INIA Quilamapu No 24 (Chillán, Chile), 31p.

Aguilera, A.; E. Cisternas, M. Gerding \& H. Rambuena. 1996. Plagas de las praderas. En Ruiz (ed.), Praderas para Chile. $2^{\mathrm{a}}$ ed. (Santiago, Chile), pp 309337.

BonNEmaison, L. 1975. Enemigos animales de las plantas cultivadas y forestales. Vol. I. Oikos Tau Edic. (Barcelona, España), 543p.

Borror, D.; Ch. Triplehorn \& M. Johnson. 1989. An introduction to the study of insects. Saunders College Publish. Printed in U.S.A. 875p.

Cisternas, E.; A. Aguilera \& M. Gerding. 1990. La pulga saltona de la alfalfa, Sminthurus viridis (L.) (Collembola: Sminthuridae), fitófago detectado en 
Chile. Agric. Técnica (Chile) 50(4):397-399.

Cisternas , E. 1994. Plagas de la alfalfa en la X Región y su control. Seminario producción y utilización de la alfalfa en la X Región. INIA serie Remehue No54 (Osorno, Chile), pp67-87.

Chinery, M. 1988. Guía de campo de los insectos de España y Europa. Ed. Omega (Barcelona), 402p.

GonZÁLEZ, R. 1989. Insectos y ácaros de importancia agrícola y cuarentenaria en Chile. Edit. Ograma, Santiago, Chile, 310p.

Greenslade, D. 1991. Collembola (Springtails). IN: The Insects of Australia. $2^{\mathrm{a}}$ edi. Vol. I, Commonwealth Scientific and Industrial Research Organization (Division of Entomology), Melbourne University Pres, pp. 252-264.

Gillot, C. 1980. Entomology. Plenum Press. New York and London, 729p.

Klein, C.\& D. Waterhouse. 2000. Distribución e importancia de los artrópodos asociados a la agricultura y silvicultura en Chile. Australian Centre for International Agricultural Research, Canberra, Australia, ACIAR Monograph $N^{\circ}$ 68, 231p.

Odum, E. 1972. Ecología. Estructura y función de la naturaleza. $3^{\mathrm{a}}$ Ed. Edit. Interamericana (México), $857 \mathrm{p}$.

Pedigo, L. 1989. Entomology and pests management:
Macmillan Publishing Company New York. Collier Macmillan Publishers (London), 645p.

Phillips, J. 1976. Fisiología Ecológica. Blume Edic. Madrid, 248p.

Prado, E. 1991. Artrópodos y sus enemigos naturales asociados a plantas cultivadas en Chile. INIA La Platina. Serie Boletín Técnico No 169 (Santiago, Chile), 203p.

Richards, O. \& R. DAVIES. 1984. Tratado de entomología IMMS. Clasificación y Biología. Vol. II. Edic. Omega (Barcelona, España), 998p.

Rouanet, J. 1983a. Clasificación agroclimática IX Región. Macroárea IV. Investigación y Progreso Agropecuario. INIA Carillanca 2(4):25-28.

RouAnet, J. 1983b. Clasificación agroclimática IX Región. Macroárea II. Investigación y Progreso Agropecuaria. INIA Carillanca 2(2):22-26.

Wallace, M. \& Y. Mackerras. 1979. The endognathus

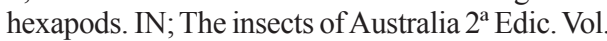
I. Commonwealth Scientific and Industrial Research Organization (Division of Entomology), Melbourne University Press, pp. 205-211.

WAYNE, D. 1988. Estadística con aplicaciones a las ciencias sociales y a la educación. Mac Graw-Hill (México), 503p.

Fecha de recepción: 28/06/04

Fecha de aceptación: 05/11/04 